\title{
Hipertensão intracraniana com manifestações oculares associada ao uso de tetraciclina: relato de caso
}

\author{
Intracranial hypertension with ocular manifestation during the use of tetracycline: \\ case report
}

Frederico Xavier dos Santos ${ }^{1}$

André Parolin²

Elissandro Márcio Silva Lindoso ${ }^{3}$

Fernando Henrique Xavier Santos ${ }^{4}$

Luciene Barbosa de Sousa ${ }^{5}$
Trabalho realizado no Hospital Oftalmológico de Sorocaba.

${ }^{1}$ Residente de oftalmologia do segundo ano do Hospital oftalmológico de Sorocaba. Sorocaba (SP).

${ }^{2}$ Médico oftalmologista graduado pela Universidade $\mathrm{Fe}$ deral de São Paulo (UNIFESP). São Paulo (SP).

${ }^{3}$ Residente de oftalmologia do primeiro ano do Hospital Oftalmológico de Sorocaba. Sorocaba (SP).

${ }^{4}$ Acadêmico do quinto ano do curso de medicina da

Pontifícia Universidade Católica de Sorocaba. Sorocaba (SP).

${ }^{5}$ Doutora em oftalmologia pela UNIFESP. São Paulo (SP). Chefe da Residência do Hospital Oftalmológico de Sorocaba. Sorocaba (SP).

Endereço para correspondência: Frederico Xavier dos Santos. Rua Bernardo Ferraz de Almeida, 187/01 Sorocaba (SP) CEP 18031-000

E-mail: fredxsantos@hotmail.com

Recebido para publicação em 10.11.2004

Versão revisada recebida em 15.02.2005

Aprovação em 14.06.2005

\begin{tabular}{|c|}
\hline RESUMO \\
\hline Os autores relatam o caso de uma paciente jovem, do sexo feminino, que \\
desenvolveu quadro de hipertensão intracraniana benigna com manifes- \\
tações oculares em concomitância ao uso de tetraciclina para o tratamento \\
de otite. Esta é uma reação adversa rara deste medicamento, descrito em \\
bula e com alguns relatos de caso em literatura internacional. \\
\hline
\end{tabular}

Descritores: Otite/quimioterapia; Tetraciclina/uso terapêutico; Tetraciclina/efeitos adversos; Hipertensão intracraniana/etiologia; Pseudotumor cerebral; Manifestações oculares; Transtornos da visão; Papiledema; Relatos de casos [tipo de publicação]

\section{INTRODUÇÃO}

As tetraciclinas são antibióticos primariamente bacteriostáticos, porém quando presentes em altas concentrações agem de maneira bactericida ${ }^{(1)}$.

O espectro de ação das tetraciclinas é praticamente o mesmo. Muitos Gram-positivos aeróbios são susceptíveis, porém atualmente há cepas de estafilococos e estreptococos (principalmente pneumococo) resistentes. É ativa contra algumas cepas de E. coli e Pseudomonas sp. ${ }^{(1)}$.

São drogas alternativas no tratamento da brucelose, infecções por Clamydia (uretrite e doença inflamatória pélvica), riquetsioses, cólera e febre recorrente. São também utilizadas em infecções por Mycoplasma pneumoniae, linfogranuloma venéreo e cancróide, bem como no tratamento da gonorréia em pacientes alérgicos a penicilina ${ }^{(1)}$.

Com relação à oftalmologia, as tetraciclinas vêm apresentando atualmente indicações mais restritas devido ao advento de novos antibióticos com maior espectro de ação e posologia mais confortável levando a uma aderência maior ao tratamento. São usadas na forma tópica para o tratamento da infecção ocular clamidiana (tracoma ativo e oftalmia neonatal clamidiana), podendo ser associado a eritromicina via oral dependendo da severidade do quadro clínico $^{(2)}$. São drogas de escolha para o tratamento da rosácea, apresentando melhora significativa das pápulas e pústulas, com efeito, menor com relação ao eritema e telangectasias ${ }^{(2)}$. A antibioticoterapia sistêmica com tetraciclina deve ser adotada para o tratamento da rosácea sendo que alguns pacientes necessitam de uma baixa dose de manutenção por períodos prolongados para prevenção de recorrência da doença ativa ${ }^{(2)}$.

Como todo antibiótico as tetraciclinas também apresentam algumas reações adversas que vão desde alterações gastrintestinais (náuseas, vômitos, e diarréia), erupção de pele, alteração na formação dentária em crianças menores de doze anos, até quadros mais raros e severos como: colite, pericardite e hipertensão intracraniana ${ }^{(1)}$. 
Este trabalho consiste na descrição de um caso de hipertensão intracraniana benigna com manifestações oculares, entre elas papiledema bilateral em concomitância ao uso de tetraciclina.

\section{RELATO DE CASO}

F. C. N., sexo feminino, branca, 22 anos de idade, estudante, natural e procedente de Viradouro - SP, procurou assistência oftalmológica em 23/09/2003 queixando-se de turvação visual em $\mathrm{AO}$, acompanhado de cefaléia holocraniana de leve intensidade e zumbidos. Associado ao quadro, a paciente relata que os olhos "entortaram".

Refere que usou tetraciclina $500 \mathrm{mg}$, via oral, de $6 / 6 \mathrm{~h}$ por três dias para tratamento de otite em meados de agosto. A medicação foi recomendada por farmacêutico. No segundo dia de uso da droga ocorreu turvação visual e no terceiro dia os olhos "entortaram". Interrompeu o uso da medicação no terceiro dia. A mesma não apresentava história prévia de hipertensão arterial sistêmica, diabetes melitus, tromboembolismo, ou mesmo uso de outro medicamento nos últimos seis meses.

Ao exame oftalmológico apresentava acuidade visual de 20/60 em OD e de 20/30 em OE, avaliado em tabela de Snellen (6 metros de distância), tonometria de aplanação $16 \mathrm{mmHg}$ em AO. À biomicroscopia não se verificou alterações, bem como nos reflexos pupilares (direto e consensual). Com relação ao exame da musculatura extrínseca ocular a paciente apresentava esotropia alternante de 30 dioptrias prismáticas avaliado pelo Krismky (Figura 1).

No exame de mapeamento de retina foi observado papiledema bilateral, sem outras alterações (Figura 2). Foi medicada com acetazolamida $\left(\right.$ Diamox $\left.^{\circledR}\right)$ de $6 / 6$ h. Encaminhada para avaliação neurológica que revelou exames geral e neurológico normais, sendo adicionado furosemida via oral ao tratamento.

Apresentava tomografia computadorizada de crânio e campimetria computadorizada (Humphrey) normais.

Após esses achados foi realizado o diagnóstico de hipertensão intracraniana benigna, relacionada ao uso de tetraciclina. No retorno em 13/10/2003, a paciente referiu que os zumbidos desapareceram e que os olhos voltaram à posição normal (Figura 3). Apresentava acuidade visual corrigida de 20/25 em AO. Na fundoscopia, o edema de papila diminuiu, porém, ainda persistia em ambos os olhos.

Em 22/11/2003, a paciente apresentava a mesma acuidade visual do último exame. Na fundoscopia, a papila se encontrava

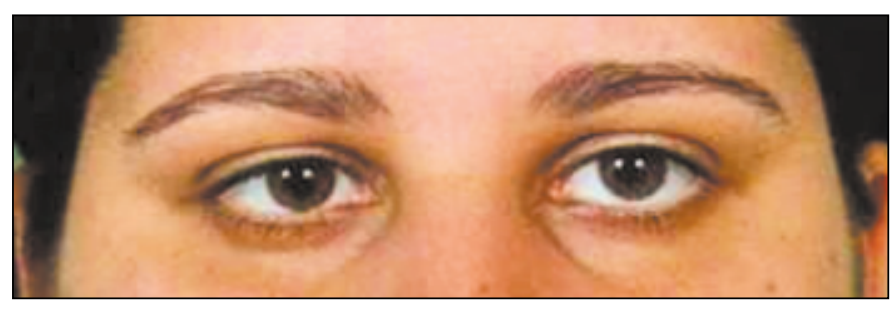

Figura 1 - Esotropia do olho direito (medindo 30 dioptrias)

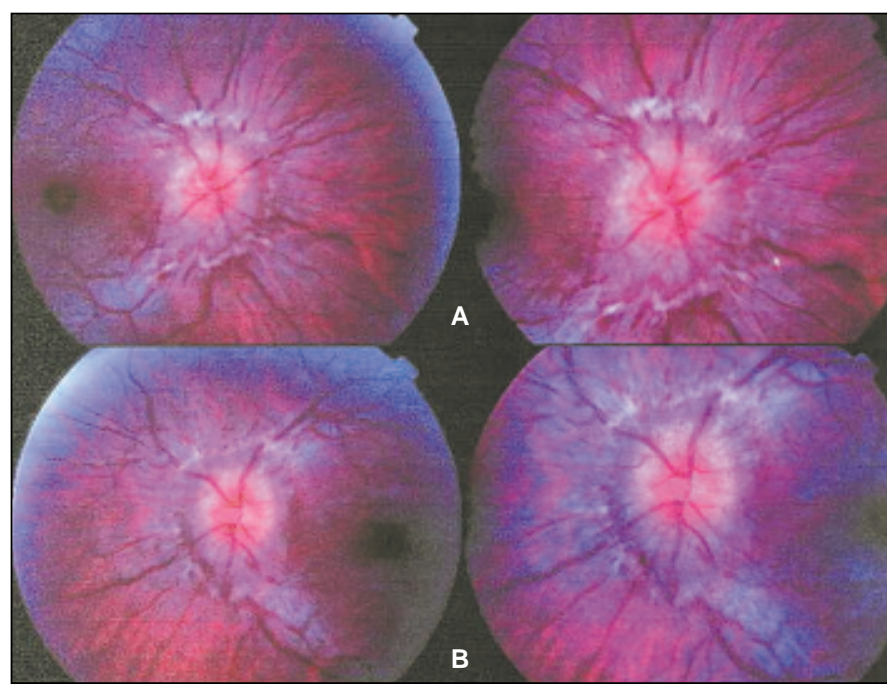

Figura 2 - Retinografia evidenciando edema de papila bilateral: $A=$ olho direito e $\mathrm{B}=$ olho esquerdo

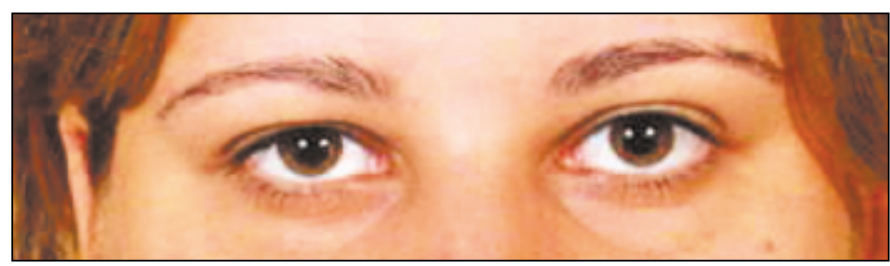

Figura 3 - Ortotropia após 5 meses de suspensão da tetraciclina

sem edema, apenas com dobras retinianas peripapilares, sendo suspensa a medicação. Em 03/02/2004, a paciente apresentava acuidade visual de 20/20p em AO com correção. Na fundoscopia apresentava olho direito sem alterações e olho esquerdo com discreta palidez de papila em quadrante temporal.

\section{DISCUSSÃO}

A hipertensão intracraniana benigna é caracterizada pela elevação da pressão intracraniana sem aumento dos ventrículos ou lesões expansivas. Essa doença está associada com mulheres jovens, obesas ou com aumento de peso recente ${ }^{(3)}$. Apresenta incidência de 1/100.000 pessoas por ano e prevalência de 19,3/100.000 em mulheres obesas entre 20 e 40 $\operatorname{anos}^{(3)}$. Os sinais e sintomas incluem dor de cabeça, embaçamento e obscuração visual transitória, perda de visão, edema do disco bilateral (papiledema) e diplopia secundária à paralisia do sexto nervo craniano. O exame neurológico é normal. Cefaléia e papiledema são as mais freqüentes manifestações ${ }^{(4)}$.

A elevação da pressão venosa intracraniana é sugerida como mecanismo universal responsável pela hipertensão intracraniana benigna de várias etiologias ${ }^{(4)}$. Isto já é bem demonstrado em determinadas condições clinicas, como: distúrbios nutricionais e endócrinos ou com uso certos medicamentos ${ }^{(5)}$. 
Através da análise do quadro clínico apresentado, associando este aos resultados dos exames complementares, bem como a exclusão de outros fatores causais para a hipertensão intracraniana, sugerimos uma forte correlação entre o quadro apresentado pela paciente com o uso de tetraciclina.

$\mathrm{Na}$ literatura não está provado que o uso da tetraciclina pode levar à hipertensão intracraniana benigna. Um estudo retrospectivo $^{(5)}$ e um estudo prospectivo ${ }^{(6)}$ envolvendo um total de 90 pacientes não encontrou associação estatisticamente significante entre tetraciclina e hipertensão intracraniana. Entretanto, nos últimos 40 anos vem sendo descritos casos que unam os dois. Gellis ${ }^{(7)}$ foi o primeiro a mencionar associação entre tetraciclina e hipertensão intracraniana em 1956, seguido por Millichap ${ }^{(8)}$ em 1959.

Após essas duas descrições iniciais, observa-se um aumento no número de relato de casos de pacientes com hipertensão intracraniana benigna supostamente associada à tetraciclina. Além de estarem presentes na literatura relatos de várias series de crianças com quadro clinico e evolução bastante sugestivos, notamos que alguns deles apresentam origem familiar, como em nove ${ }^{(9-17)}$ relatos de casos familiares de hipertensão intracraniana associado ao uso de tetraciclina, envolvendo dezenove pacientes com quadro característico, incluindo dois irmãos monozigotos, mãe-filha e mãe-filho, sugerindo a possibilidade de uma susceptibilidade genética estar presente para o desenvolvimento de hipertensão intracraniana com uso de tetraciclina.

Ainda há necessidade de mais estudos que comprovem a relação da tetraciclina na gênese da hipertensão intracraniana, porém na abordagem de pacientes com quadros semelhantes é importante realizar uma investigação um pouco mais detalhada com relação à medicação utilizada pelo paciente já que a simples suspensão da droga pode levar a resolução completa do quadro.

A hipertensão intracraniana benigna associada ao uso de tetraciclina é um quadro raro acometendo com mais freqüência mulheres jovens com sobrepeso. Sempre deve ser lembrado como diagnóstico diferencial em pacientes com manifestações clínicas de hipertensão intracraniana sem alterações anatômicas.

Frente a esses pacientes devemos realizar anamnese completa e exame oftalmológico cuidadoso para a realização de diagnóstico preciso com adoção da terapêutica adequada levando à resolução do quadro.

\section{ABSTRACT}

The authors describe a young female patient with intracranial hypertension associated with ocular manifestations, during treatment with tetracycline. This is a rare adverse effect described in the medication warnings, and in a few reported cases in the scientific literature.

Keywords: Otitis/drug therapy; Tetracycline/therapeutic use; Tetracycline/adverse effects; Intracranial hypertension/etiology; Pseudotumor cerebri; Eye manifestations; Vision disorders; Papilledema; Case reports [publication type]

\section{REFERÊNCIAS}

1. Gilman GA. As bases farmacológicas da terapêutica. 10a ed. Rio de Janeiro: Guanabara Koogan; 2003.

2. Belfort Jr R, José NK. Córnea clínica-cirúrgica. São Paulo: Roca;1997.

3. Durcan FJ, Corbett JJ, Wall M. The incidence of pseudotumor cerebri; population studies in Iowa and Louisiana. Arch Neurol. 1988;45(8):875-7.

4. Giuseffi V, Wall M, Siegel PZ, Rojas PB. Symptoms and disease associations in idiopathic intracranial hypertension (pseudotumor cerebri): a case-control study. Neurology. 1991;41(2 Pt 1):239-44.

5. Karahalios DG, Rekate HL, Khayata MH, Apostolides PJ. Elevated intracranial venous pressure as a universal mechanism in pseudotumor cerebri of variying etiologies. Neurology. 1996;46(1):198-202.

6. Ireland B, Cobertt J, Wallace RB. The search for causes of idiopathic intracranial hypertension. A preliminary case-control study. Arch Neurol. 1990;47(3): 315-20.

7. Gellis S. Year book of Pediatrics - 1956 to 1957. Chicago: Year Book; 1956.

8. Millichap JG. Benign intracranial hypertension and otitic hydrocephalus. Pediatrics. 1959;23(2):257-9.

9. Buchheit WA, Burton C, Haag B, Shaw D. Papilloedema and idiopathic intracranial hypertension. N Engl J Med. 1969;280(17):938-42.

10. Siegel NJ, Spackman TJ. Chronic hypervitaminosis A with intracranial hypertension and low cerebrospinal fluid concentration of protein. Two illustrative cases. Clin Pediatr (Phila). 1972;11(10):580-4.

11. Howe JG, Saundes M Clarke PRR. Familial benign intracranial hypertension. Acta Neurochir (Wien). 1973;29(2):173-5.

12. Venable HP. Pseudo-tumor cerebri: further studies. J Natl Med Assoc. 1973; 65(3):194-7.

13. Rothner AD, Brust JC. Pseudotumor cerebri.: report of a familial occurrence. Arch Neurol. 1974;30(1):110-1.

14. Mikkelsen B, Ehlers N, Thomsen HG. Vitamin A intoxication causing pa pilledema and simulating acute encephalitis. Acta Neurol Scand. 1974;50(5): 642-50.

15. Traviesa DC, Schwartzman RJ, Glaser JS, Savino P. Familial benign intracranial hypertension. J Neurol Neurosurg Psichiatry. 1976;39(5):420-3.

16. Shapiro I, Shapiro SK. Familial pseudotumor cerebri and the empty sella syndrome. Ann Ophthalmol. 1980;12(9):1045-8.

17. Coffey CE, Ross DR, Massey EW, Olanow CW. Familial benign intracranial hypertension and depression. Can Neurol Sci. 1982;9(1):45-7.

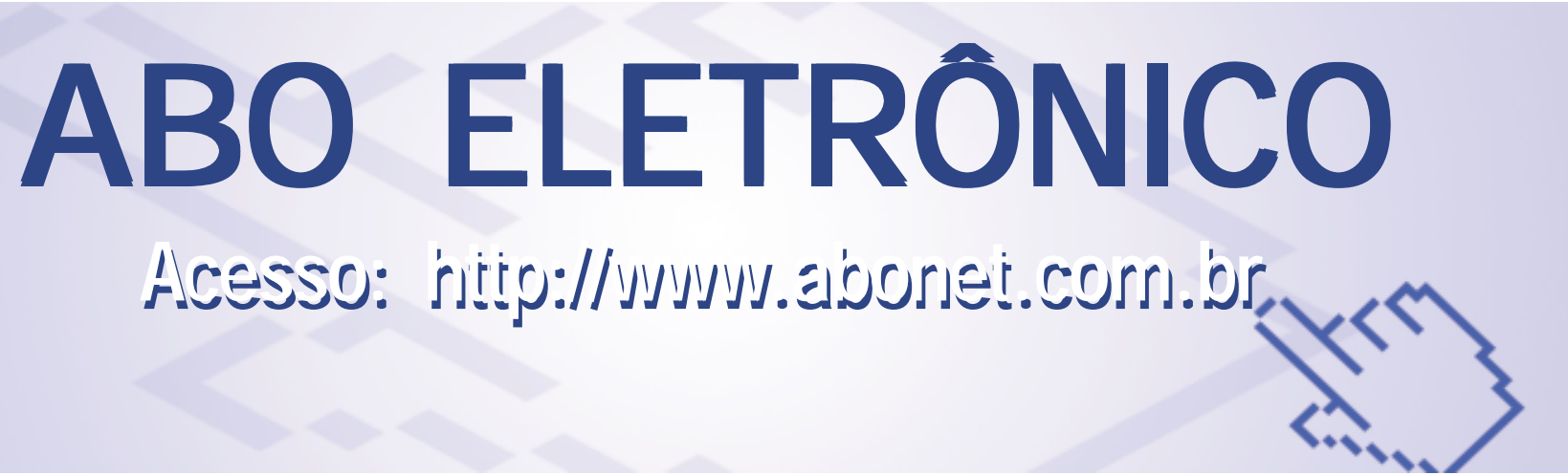

